Case Reports in
Gastroenterology
Case Rep Gastroenterol 2020;14:510-515

DOI: 10.1159/000508861

Published online: October 22, 2020

(C) 2020 The Author(s)

Published by S. Karger AG, Basel www.karger.com/crg

This article is licensed under the Creative Commons Attribution-NonCommercial 4.0 International License (CC BY-NC) (http://www.karger.com/Services/OpenAccessLicense). Usage and distribution for commercial purposes requires written permission.

\title{
Barrett's Esophageal Adenocarcinoma Involving a White Globe Appearance within the Long-Segment Barrett's Esophagus
}

\author{
Yo Kubota ${ }^{a}$ Satoshi Tanabe ${ }^{b}$ Yohei Harada ${ }^{a}$ Seigo Nakatani ${ }^{a}$ \\ Yasuaki Furue $^{a}$ Takuya Wada $^{a}$ Akinori Watanabe ${ }^{a}$ Kenji Ishido $^{a}$ \\ Chikatoshi Katada ${ }^{a}$ Wasaburo Koizumia \\ aDepartment of Gastroenterology, Kitasato University School of Medicine, \\ Sagamihara, Japan; ${ }^{b}$ Department of Advanced Medicine Research and Development \\ Center for New Medical Frontiers, Kitasato University School of Medicine, \\ Sagamihara, Japan
}

\section{Keywords}

Barrett's esophageal adenocarcinoma - Intraglandular necrotic debris · White globe appearance

\begin{abstract}
The diagnosis of Barrett's esophageal adenocarcinoma (BEA) in patients with Barrett's esophagus (BE) using endoscopy can be difficult and there are few specific endoscopic findings for BEA. However, white globe appearance (WGA) has been reported to be a specific endoscopic finding for early gastric cancer. We encountered a 51-year-old male patient with BEA exhibiting WGA. Esophagogastroduodenoscopy identified a red, depressed lesion of $10 \mathrm{~mm}$ within the long-segment $B E(L S B E)$, while magnifying endoscopy with narrow-band imaging identified WGA. Endoscopic submucosal dissection (ESD) was performed based on our suspicion of BEA. Based on the ESD findings, we diagnosed adenocarcinoma accompanying LSBE histopathologically. WGA was identified, and intraglandular necrotic debris was discovered histologically at the same site. Therefore, WGA may be helpful in the diagnosis of BEA.
\end{abstract}




\section{Case Reports in Gastroenterology}

Case Rep Gastroenterol 2020;14:510-515

DOI: $10.1159 / 000508861$

(c) 2020 The Author(s). Published by S. Karger AG, Basel www.karger.com/crg

Kubota et al.: Barrett's Esophageal Adenocarcinoma Involving a White Globe Appearance

\section{Introduction}

Barrett's esophagus (BE) is characterized by the transformation of esophageal squamous cell epithelium to metaplastic columnar epithelium with goblet cells, and it is recognized as a precursor lesion to Barrett's esophageal adenocarcinoma (BEA) [1]. The incidence is increasing in most Western populations [2], and the prognosis for patients with BEA is strongly related to the stage at diagnosis [3]. Therefore, surveillance for BEA in BE patients is considered important for the realization of early therapeutic intervention for BEA. Biopsy of a suspected lesion site and random four-quadrant biopsies along with regular esophagogastroduodenoscopy (EGD) are recommended as a means of surveillance for BEA in patients with BE [4]. In recent years there have been improvements in diagnostic technology, such as the proposal of the Barrett's International NBI Group (BING) criteria using magnifying endoscopy with narrow-band imaging (M-NBI) [5]. However, difficulties in diagnosing BEA are still encountered in many cases. White globe appearance (WGA), which is defined as "a white, spherical appearance that is $1 \mathrm{~mm}$ or less in size, found just below the epithelium," is identified using M-NBI and is considered to be a specific endoscopic finding for early gastric cancer (EGC) [6, 7]. WGA in BEA patients is very rare and has been reported in only two previous cases [8]. In this article we provide a relevant literature review and report on our experience of a patient who developed BEA with WGA in the long-segment BE (LSBE) over the course of long-term clinical course monitoring, for whom curative resection of the lesion was achieved by endoscopic submucosal dissection (ESD).

\section{Case Report}

The patient was a 51-year-old man. EGD performed 6 years before at another hospital revealed erosion in the LSBE and he was hospitalized in the same year. EGD performed in our hospital also showed LSBE measuring 27-35 cm, and an erosion site measuring $5 \mathrm{~mm}$ was observed in the LSBE (Fig. 1a-c). On biopsy, this was determined to be a glandular abnormality, and follow-up EGD was performed annually in accordance with the treatment policy. In addition, as the patient exhibited heartburn symptoms, he continued to receive oral proton pump inhibitor treatments. Despite undergoing EGD and biopsy every year, it was not possible to point out any clear malignant findings (Fig. 1d-h). Six years later, EGD revealed a depressed, reddish lesion measuring approximately $10 \mathrm{~mm}$ at the same site (Fig. 2a). A demarcation line, an irregular microsurface pattern, and an irregular microvascular pattern were observed on M-NBI (Fig. 2b). In addition, we observed a white spherical lesion suspected of being WGA close to the demarcation line (Fig. 2c). Although biopsy revealed no malignant findings, based on the M-NBI findings and the presence of WGA, we determined that the lesion was highly likely to be BEA, and for this reason ESD was performed on the same site using an IT-knife 2 (KD-611L; Olympus Medical Systems Co., Ltd, Tokyo, Japan) device. The procedure was completed without any complications.

Histopathological examination of the ESD specimen revealed that the background mucosa was BE with glandular epithelium, with a well-differentiated (tub1) adenocarcinoma $8 \times 6 \mathrm{~mm}$ in size with a macroscopic classification of 0 -IIc, and that the lesion was confined to the superficial muscularis mucosa, with no evidence of vascular invasion (Fig. 3a-d). In addition, histologically we found intraglandular necrotic debris (IND) presenting as a profoundly dilated duct with eosinophilic material and necrotic epithelial fragments consistent with the WGA observed by endoscopy (Fig. 3e, f). Based on the above findings, the patient was diagnosed with 


\section{Case Reports in Gastroenterology}

Case Rep Gastroenterol 2020;14:510-515

DOI: $10.1159 / 000508861$

(c) 2020 The Author(s). Published by S. Karger AG, Basel www.karger.com/crg

Kubota et al:: Barrett's Esophageal Adenocarcinoma Involving a White Globe Appearance

BEA accompanied by WGA. No recurrence has occurred since he underwent ESD at the time of the publication of this report.

\section{Discussion}

On this occasion, we diagnosed BEA with WGA using M-NBI. Yoshida et al. [7] reported that the accuracy, sensitivity, and specificity of the WGA diagnosis to detect gastric cancer are $69.1,21.4$, and $97.5 \%$, respectively. Therefore, WGA is regarded as a specific endoscopic finding for EGC. Further, in EGC, WGA was often located close to the demarcation line between the cancerous mucosa and the surrounding mucosa [6]. In addition IND, defined as eosinophilic material with necrotic epithelial fragments in the lumen of dilated glands, is regarded as a histological marker for the detection of high-grade dysplasia or invasive carcinoma of the stomach, with a sensitivity and specificity of detection of 43.1 and $98.7 \%$, respectively [6, 9]. Moreover, necrotic debris is thought to be recognized as white in color because it strongly scatters and reflects the light projected from the endoscope; as such, WGA corresponds to IND in the histopathological examination [6]. However, Iwamuro et al. [10] pointed out the presence of WGA in the noncancerous stomach and reported the possibility of acid secretion inhibitors, other pharmacotherapies, and Helicobacter pylori infection being the cause of WGA. It is supposed that parietal cell outgrowth and basal gland cysts are caused by hypergastrinemia associated with long-term oral use of acid secretion inhibitors, and that fluid inside the basal gland cysts is recognized as a white spherical appearance. For this reason, it is important to combine WGA findings with other endoscopic findings to reach a diagnosis.

The present patient had a stomach negative for H. pylori and had been taking an oral proton pump inhibitor for esophageal hiatal hernia and gastroesophageal reflux disease over a long period of time. Although it can be suggested that basal gland cysts may have formed with proton pump inhibitor use, we concluded the identification of WGA in BEA based on the endoscopic observation of white spherical lesions close to the demarcation line, and histological observation of profoundly dilated ducts with eosinophilic material and necrotic epithelial fragments. BEA accompanied by WGA has only been reported in 2 cases to date, both by Tonai et al. [8]. We therefore believe that the rarity of this case makes it valuable. Although biopsies did not reveal any malignant findings for the present patient, we performed ESD based on the M-NBI and WGA findings and were able to reach a definitive diagnosis of BEA. For this reason, we believe that identification of WGA contributed sufficiently to diagnosing BEA.

These results suggest that WGA with IND may serve as a specific endoscopic finding for EGC as well as for BEA. Further cases must be studied in order to verify these findings.

\section{Statement of Ethics}

The authors confirm that the patient provided written informed consent to publish the case report as well as the pictures.

\section{Conflict of Interest Statement}

The authors have no conflicts of interest to declare. 


\section{Case Reports in Gastroenterology}

\section{Funding Sources}

This work did not receive any kind of financial support from any source in the public, commercial, or not-for-profit sectors.

\section{Author Contributions}

Y. Kubota, S. Tanabe, Y. Harada, S. Nakatani, Y. Furue, T. Wada, A. Watanabe, K. Ishido, and C. Katada interpreted the patient's imaging and discussed the treatment plan. Y. Kubota and S. Tanabe drafted the manuscript. S. Tanabe and W. Koizumi gave the final approval of the version to be published. All authors read the manuscript and approved it for publication.

\section{References}

1 Nelsen EM, Hawes RH, Iyer PG. Diagnosis and management of Barrett's esophagus. Surg Clin North Am. 2012 Oct;92(5):1135-54.

2 Thrift AP. Barrett's esophagus and esophageal adenocarcinoma: how common are they really? Dig Dis Sci. 2018 Aug;63(8):1988-96.

3 Qumseya B, Sultan S, Bain P, Jamil L, Jacobson B, Anandasabapathy S, et al.; ASGE Standards of Practice Committee; ASGE Standards of Practice Committee Chair. ASGE guideline on screening and surveillance of Barrett's esophagus. Gastrointest Endosc. 2019 Sep;90(3):335-359.e2.

4 Provenzale D, Schmitt C, Wong JB. Barrett's esophagus: a new look at surveillance based on emerging estimates of cancer risk. Am J Gastroenterol. 1999 Aug;94(8):2043-53.

5 Sharma P, Bergman JJ, Goda K, Kato M, Messmann H, Alsop BR, et al. Development and Validation of a Classification System to Identify High-Grade Dysplasia and Esophageal Adenocarcinoma in Barrett's Esophagus Using Narrow-Band Imaging. Gastroenterology. 2016 Mar;150(3):591-8.

6 Doyama H, Yoshida N, Tsuyama S, Ota R, Takeda Y, Nakanishi H, et al. The "white globe appearance" (WGA): a novel marker for a correct diagnosis of early gastric cancer by magnifying endoscopy with narrow-band imaging (M-NBI). Endosc Int Open. 2015 Apr;3(2):E120-4.

7 Yoshida N, Doyama H, Nakanishi H, Tsuji K, Tominaga K, Matsunaga K, et al. White globe appearance is a novel specific endoscopic marker for gastric cancer: A prospective study. Dig Endosc. 2016 Jan;28(1):59-66.

8 Tonai Y, Ishihara R, Yamasaki Y, Kanesaka T, Yamamoto S, Akasaka T, et al. First reports of esophageal adenocarcinoma with white globe appearance in Japanese and Caucasian patients. Endosc Int Open. 2016 Oct;4(10):E1075-7.

9 Watanabe Y, Shimizu M, Itoh T, Nagashima K. Intraglandular necrotic debris in gastric biopsy and surgical specimens. Ann Diagn Pathol. 2001 Jun;5(3):141-7.

10 Iwamuro M, Tanaka T, Sakae H, Yamasaki Y, Kanzaki H, Kawano S, et al. Two cases of white globe appearance in non-cancerous stomach. Ecancermedicalscience. 2018 Aug;12:856. 


\section{Case Reports in Gastroenterology}

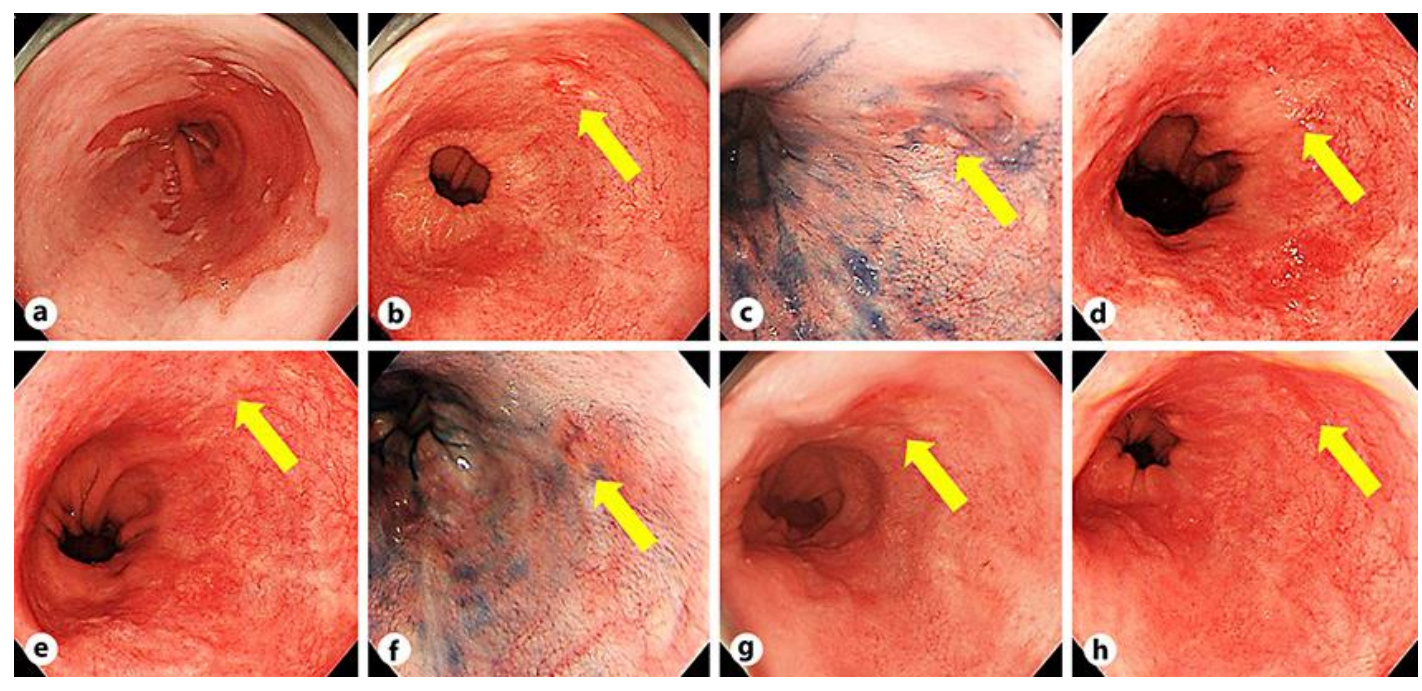

Fig. 1. a An LSBE measuring $8 \mathrm{~cm}$ in length was observed. b White light imaging revealed an erosion, $5 \mathrm{~mm}$ in diameter, in the LSBE (arrow). c Indigo carmine staining showing the border of the lesion (arrow). $\mathbf{d}$ One year later (arrow). e Two years later (arrow). $\mathbf{f}$ Three years later (arrow). $\mathbf{g}$ Four years later (arrow). $\mathbf{h}$ Five years later (arrow). LSBE, long-segment Barrett's esophagus.
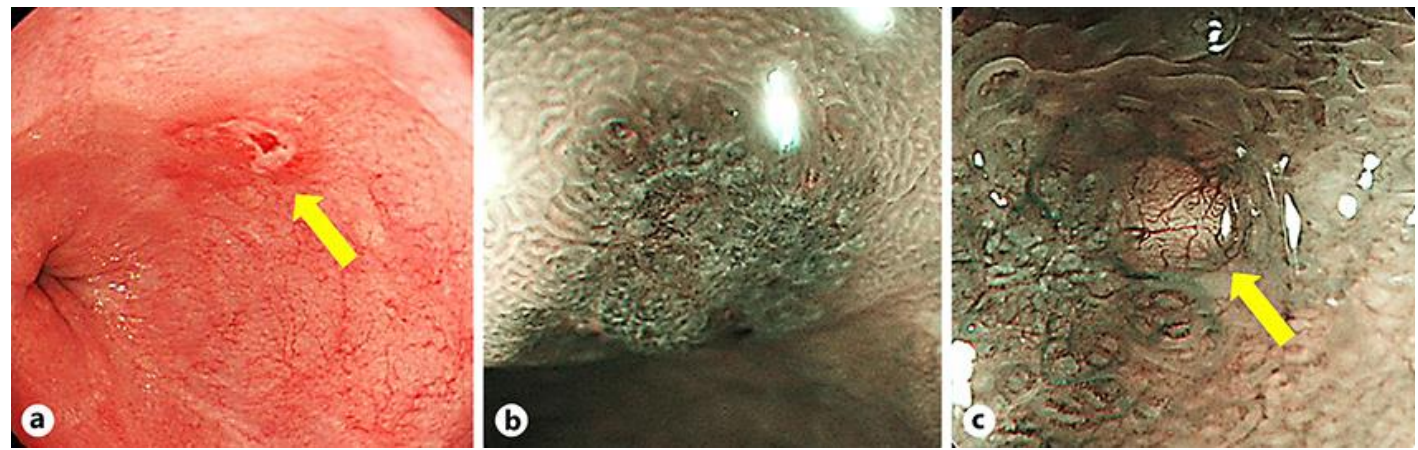

Fig. 2. a In white light imaging a slightly red, depressed lesion, $10 \mathrm{~mm}$ in diameter, was revealed in the LSBE (arrow). b M-NBI revealed a brownish area. c Using M-NBI, demarcation line, irregular microsurface pattern, and irregular microvascular pattern were revealed in the lesion. WGA was revealed near the demarcation line (arrow). LSBE, long-segment Barrett's esophagus; M-NBI, magnifying endoscopy with narrow-band imaging; WGA, white globe appearance. 
Case Reports in Gastroenterology
Case Rep Gastroenterol 2020;14:510-515

DOI: 10.1159/000508861

(c) 2020 The Author(s). Published by S. Karger AG, Basel www.karger.com/crg

Kubota et al.: Barrett's Esophageal Adenocarcinoma Involving a White Globe Appearance
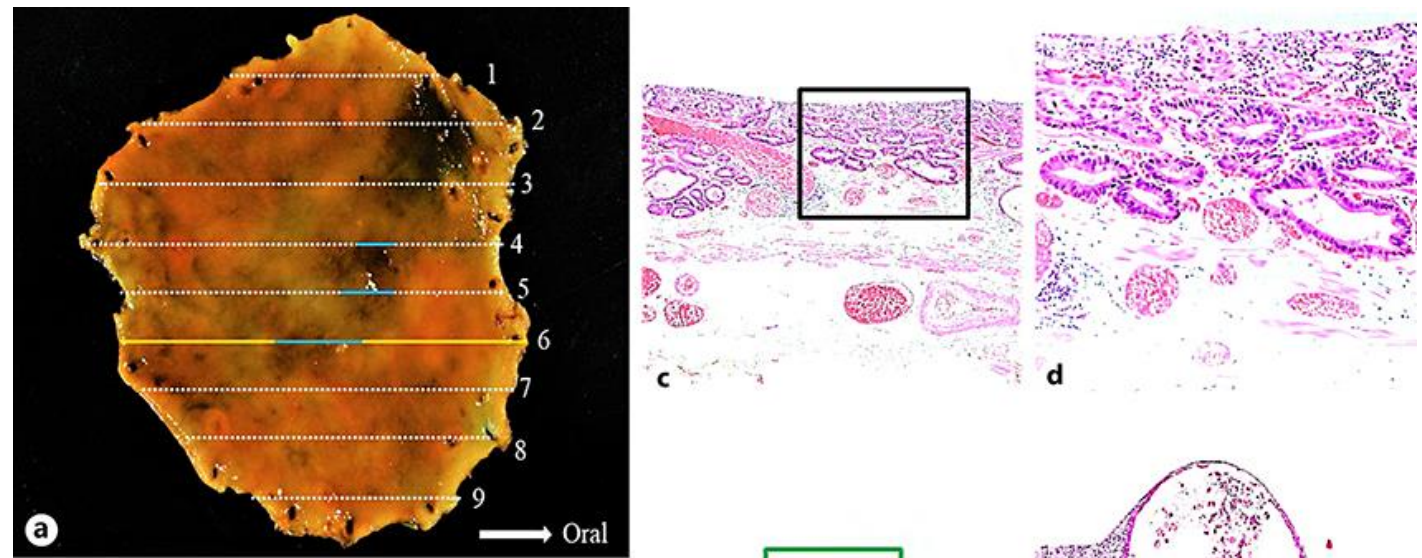

b

No.6

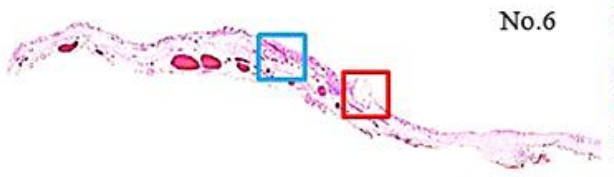

Fig. 3. a ESD specimen. b Low-power view of specimen No. 6 showed that the tumor was limited to the superficial muscularis mucosa, without lymphovascular involvement (yellow square). c High-power view of specimen No. 6 , showed the findings of BE (hematoxylin and eosin staining, $\times 40$ ) (blue square). $\mathbf{d}$ Magnified view of $\mathbf{c}$ (hematoxylin and eosin staining, $\times 100$ ) (black square). e A dilated neoplastic gland with eosinophilic material and necrotic epithelial fragments typical of IND were revealed. This finding was identified at the site of WGA (hematoxylin and eosin staining, $\times 40$ ) (red square). $f$ Magnified view of $\mathbf{e}$ (hematoxylin and eosin staining, $\times 100$ ) (green square). BE, Barrett's esophagus; ESD, endoscopic submucosal dissection; IND, intraglandular necrotic debris; WGA, white globe appearance. 